\title{
ARTICLE
}

\section{Neutron fluence analyses around the reactor pressure vessel of BWR using MCNP with a heterogeneous and homogeneous mixed core model}

\author{
Kaoru Matsushita $^{\mathrm{a}^{*}}$ and Masahiko Kurosawa ${ }^{\mathrm{a}}$ \\ ${ }^{a}$ Toshiba Corporation, 8, Shinsugita-cho, Isogo-ku, Yokohama, Kanagawa-ken, 235-8523, Japan
}

\begin{abstract}
For reactor pressure vessel (RPV) material surveillance program, it is necessary to obtain fast neutron fluence. In this work, Monte Carlo transport code MCNP is applied to analyze it for a Boiling Water Reactor (BWR) with a heterogeneous and homogeneous mixed core model (HHMCM) and its applicability is examined. The analyses of using MCNP with HHMCM are performed to obtain the neutron flux and the reaction rate of the dosimeter wires at the inner surface of the RPV of an existing 800 MWe BWR plant in Japan. As a result, the neutron flux and the reaction rates can be estimated with an uncertainty of $8 \%$ at most. In addition, HHMCM can reduce a calculation time to $1 / 9$ compared with a case of all bundles treated as heterogeneous.
\end{abstract}

Keywords: neutron flux; reaction rate; MCNP; BWR; reactor pressure vessel

\section{Introduction}

For RPV material surveillance program, it is necessary to obtain neutron fluence at the RPV to estimate its neutron irradiation embrittlement etc., and the neutron flux is usually analyzed using two-dimensional discrete ordinates code such as DORT [1] for Japanese plants. Table 1 shows the calculation conditions using DORT which was applied to an existing 800 MWe BWR plant in Japan. As shown in this table, a multi-group energy nuclear transport cross-section and spectrum with a homogeneous core model were used. The calculation accuracy of the fast neutron flux $(\mathrm{E} \geq 0.1 \mathrm{MeV})$ was evaluated at approximately $30 \%$ comparing the measured reaction rate of the dosimeter wire with the calculated one.

On the other hand, MCNP [2] treats an arbitrary three-dimensional configuration of materials; a core can be heterogeneously-consisted of fuel rods, water rods, channel boxes, etc. MCNP can also treat a continuous energy spectrum and nuclear data library, so it could be expected to analyze the neutron flux at the RPV with a higher calculation accuracy than DORT. However, if the source region geometry is too complicated, MCNP requires a huge computing time for the source position sampling procedure. ${ }^{1}$

In this work, MCNP was applied to the neutron flux analyses at the inner surface of the RPV of the 800 MWe BWR plant, which was performed using DORT with the calculation condition in Table 1. But to reduce the huge computing time, a heterogeneous and homogeneous mixed core model (HHMCM) was applied

*Corresponding author. Email: kaoru2.matsushita@toshiba.co.jp to the calculation. HHMCM has two core regions; one is a heterogeneous region with a fuel bundle structure in detail, and another is a homogenous one which is similar to the core model using DORT. First, how to divide the core region with $\mathrm{HHMCM}$ will be examined using MCNP. Next, HHMCM will be applied to the neutron flux analyses as the same using DORT mentioned above. Finally, the calculation accuracy of HHMCM will be verified through the comparison of the neutron flux and reaction rate of the neutron dosimeter wires.

Table 1. Calculation conditions for 800 MWe BWR using DORT in the reference analysis

\begin{tabular}{ll}
\hline Item & Condition \\
\hline Geometry & $\mathrm{R}-\mathrm{R} / \mathrm{R}-\theta$ \\
Code & $\mathrm{DORT}$ \\
Order of scattering expansion & $\mathrm{P}_{5}$ \\
Number of directions & $\mathrm{S}_{12}(192)$ \\
Cross section library & MATXLIB-J33 [3] \\
Neutron energy spectrum & (collapsed 26 energy group) \\
\hline
\end{tabular}

\section{Examination of heterogeneous core range}

In this section, to determine the range of the heterogeneous fuel bundle model in HHMCM, the contributions of the fast neutron flux from each region which are heterogeneous or homogeneous to an estimation point will be examined using MCNP.

\section{1. $3 \times 10$ bundles model}

Figure 1 shows the basic concept of the divided core into regions which is usually used for the neutron flux analyses of RPV material surveillance program to 
consider exposure distributions, void distributions and fuel bundle structures etc. Region-1 has the most peripheral bundles and Region-2 is next to Region-1. The neutron flux contributions from both the regions are considered to be significant in those analyses. Region-3 and Region-4 are the remaining regions which are divided into two regions to have approximately the same equivalent radius. Based on this concept, the neutron flux is evaluated by two simplified models to determine the heterogeneous fuel bundle region. One is Model-1 shown on the left side in Figure 2, which consists of the heterogeneous fuel bundle model with 3 rows and 10 columns, and both a shroud and a RPV are modeled as flat plates for simplification but the distances of these structures from the fuel are the same as the $800 \mathrm{MWe}$ BWR plant in section 1. The first row in Figure 2 corresponds to Region-1 in Figure 1, the second to Region-2 and the third to Region-3. Although there are several fuel bundle types in the core, the most loaded type in Region-1 is selected as a representative for whole region because it is usually assumed that the fuel bundles in Region-1 have the most influence on the neutron flux at the RPV in Figure 2. Another is Model-2 shown on the right side in Figure 2, and the third row of Model-1 is replaced by a homogeneous region.

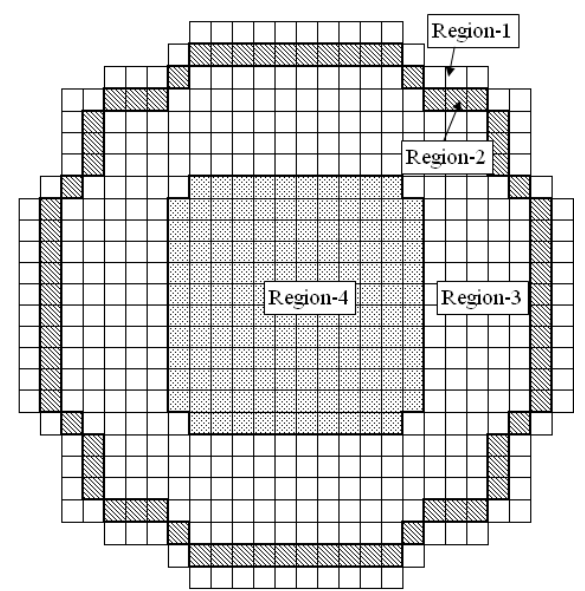

Figure 1. Fuel bundle configuration of representative 800 MWe class core

Watt fission spectrum with default parameters [2] was used and the neutron generation number distribution was considered by each node with axial distribution of bundle averaged cumulative burn-up. Neutrons were generated by each rod for the heterogeneous bundles for both Model-1 and -2 , but they were generated for the whole area of the homogeneous region of Model-2 conserving the neutron generation number. Void fraction for all regions was assumed to be $40 \%$ which corresponds to the void fraction around the center of the effective core height.

The calculation was performed with the nuclear data library based on ENDF/B-VII [2] for both models, and the point detector was set at the inner surface of the RPV as shown in Figure 2.

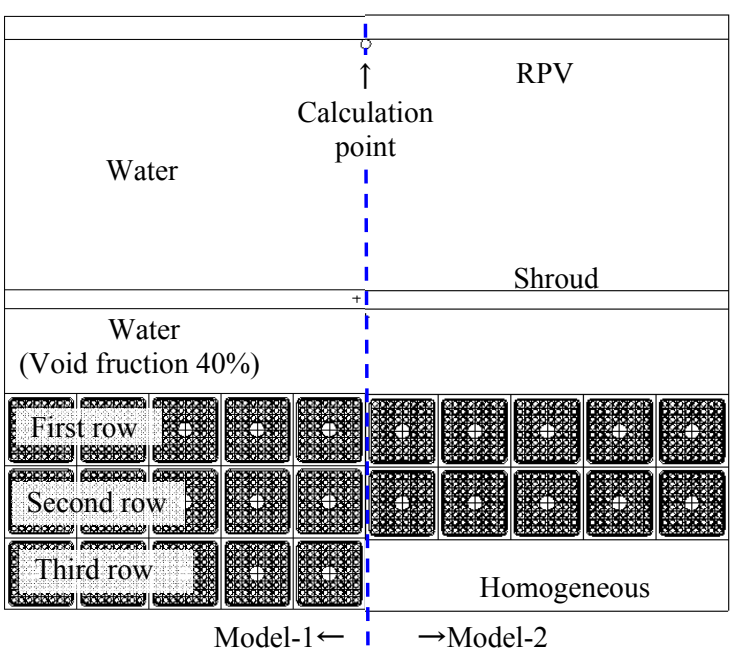

Figure $2.3 \times 10$ bundles model

\subsection{Results and discussion}

The contributions of the fast neutron flux $(E \geq 0.1$ $\mathrm{MeV}$ ) from each row are presented in Table 2, because the neutron flux at interest is for the surveillance program. In Model-1 results in Table 2, it can be seen that the main contributions to the neutron flux at the calculation point are the first and the second rows and the contribution rates reach to $94 \%$ of the total neutron flux. It can also be seen that the contribution decreases by about $30 \%$ through one row. In Model- 2 results, the contribution of the third row decreases by $12 \%$ compared with Model-1. In these results, for the third row, there is not so much of a difference in the contribution between Model-1 and Model-2 compared with total neutron flux. Furthermore, these results show if there was the fourth row, its contribution would be negligible compared with the first and second rows.

Therefore, in the estimation of the neutron flux and reaction rates of the neutron dosimeter wires at the RPV, a detail heterogeneous fuel bundle structure model is applied to the most peripheral bundles and the next to them. And a homogeneous one like the analyses by DORT is applied to the remaining region to reduce the computing time for the source position sampling procedure.

Table 2. The contributions of the neutron flux from each row

\begin{tabular}{|c|c|c|c|c|c|}
\hline \multirow{2}{*}{ Row } & \multicolumn{2}{|c|}{ Neutron flux $\left(\mathrm{n} / \mathrm{cm}^{2} / \mathrm{sec}\right)$} & \multirow{2}{*}{$\begin{array}{c}\text { Ratio of } \\
\text { total } \\
\text { (Model-1) }\end{array}$} & \multirow{2}{*}{$\begin{array}{l}\text { Ratio of } \\
\text { front row } \\
\text { (Model-1) }\end{array}$} & \multirow{2}{*}{$\begin{array}{l}\text { Model-1 } \\
\text { /Model-2 }\end{array}$} \\
\hline & Model-1 & Model-2 & & & \\
\hline 1 st & $8.6 \mathrm{E}-10$ & $8.6 \mathrm{E}-10$ & $72 \%$ & - & - \\
\hline 2nd & $2.6 \mathrm{E}-10$ & $2.6 \mathrm{E}-10$ & $22 \%$ & 0.30 & - \\
\hline $3 \mathrm{rd}$ & $7.5 \mathrm{E}-11$ & $6.7 \mathrm{E}-11$ & $6 \%$ & 0.29 & 1.12 \\
\hline total & $1.2 \mathrm{E}-09$ & $1.2 \mathrm{E}-09$ & - & - & - \\
\hline
\end{tabular}




\section{Application of HHMCM}

\subsection{Range of simulation geometry}

In this section, the fast neutron flux and the reaction rates of the neutron dosimeter wires $(\mathrm{Fe}, \mathrm{Cu})$ at the RPV inner surface of the 800 MWe BWR plant will be estimated using MCNP with HHMCM, and the accuracy of the analyses will be also verified. Figure 3 shows a calculation model consisted of HHMCM. Because of the calculation time-saving, this model was confined the simulated area which would have influence on the neutron flux at the position of the neutron dosimeter wires as shown below.

1. Let "d" be the distance from the position of the neutron dosimeter wires to the bundles which was closest to it (A, in Figure 3), and the bundles located within $\mathrm{d}+70 \mathrm{~cm}$ distance from the neutron dosimeter wires was simulated because it was judged that fast neutron flux could be attenuated by about $1 / 1000$ through $70 \mathrm{~cm}$ of reactor water.

2. A four-bundle width from the closest bundle " $A$ " to the opposite side of the neutron dosimeter wires was simulated. According to the previous section, as the fast neutron flux reduces to about $30 \%$ through one bundle width, it will reduce to about $1 / 100$ through the four bundles.

The neutron spectrum and distribution for the fixed source were treated with similar way in the previous section. Unlike the previous examination model, neutrons were generated from 5 to 20 nodes out of 24 nodes of the fuel bundles because the distances from 1 to 4 nodes and 21 to 24 nodes of the most peripheral bundles to the neutron dosimeter wires were at least greater than $70 \mathrm{~cm}$ and their contributions could be ignored compared with 5 to 20 nodes of the closest bundle "A" in Figure 3. In addition, the axial void fraction distribution was set for each region in Figure 1.

As neutron dosimeter wires are set in a holder at the RPV inner surface, track length estimator (F4) was set at the RPV inner surface as shown in Figure.3. Tally Multiplier Cards (FM4) were also used to estimate the reaction rates for $\mathrm{Cu}$ and $\mathrm{Fe}$.

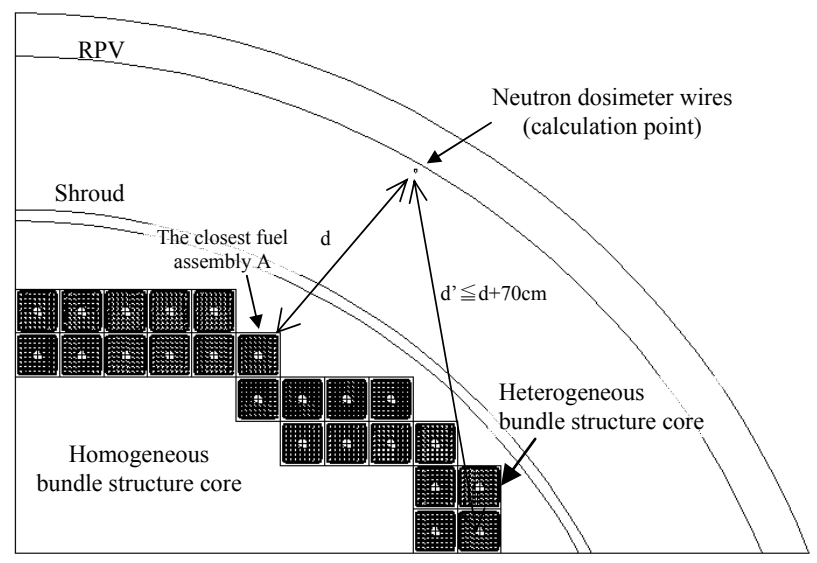

Figure 3. Calculation model of the existing $800 \mathrm{MWe}$ BWR plant with HHMCM

\subsection{Results and discussion}

\subsubsection{Neutron flux}

The ratio of the fast neutron flux $(\mathrm{E} \geq 0.1 \mathrm{MeV})$ using MCNP with HHMCM to the value based on measurement is presented in Table 3. In this table, the result using DORT for the references plant mentioned in section 1 is also shown as a comparison. As shown in Table 3, it is found that MCNP calculation with HHMCM is far more precise than DORT. However, in fact, as the measurement in Table 3 means a value with adequate weighting coefficients [3], obtained by the neutron flux calculated by DORT, it is not an exact measurement value. The estimation based on the reaction rates is more realistic to verify the accuracy of the results of using MCNP with HHMCM.

\subsubsection{Reaction rate}

The ratios of the reaction rates for $\mathrm{Fe}$ and $\mathrm{Cu}$ of using MCNP with HHMCM to measurement are shown in Table 3. The results of using DORT are also presented. It is found that the calculation of the reaction rates by MCNP with HHMCM could estimate very precisely, however the accuracy is different among Fe and $\mathrm{Cu}$. It is caused by the neutron energy threshold of each reaction, and ${ }^{54} \mathrm{Fe}(\mathrm{n}, \mathrm{p}){ }^{54} \mathrm{Mn}$ and ${ }^{63} \mathrm{Cu}(\mathrm{n}, \alpha){ }^{60} \mathrm{Co}$ are caused by the neutron of which threshold energies are greater than about $0.7 \mathrm{MeV}$ and $2 \mathrm{MeV}$ each. So, in this estimation, the calculation for the neutron flux over $0.7 \mathrm{MeV}$ is more precise than $0.7 \mathrm{MeV}$ to $2 \mathrm{MeV}$.

The accuracy of the reaction rates is better than that of the neutron flux as shown in Table 3. It is thought to be aftereffects of the measurement corrected by DORT calculation results mentioned above.

Table 3. Ratios of calculation values to measurement values in neutron flux and reaction rates

\begin{tabular}{cccc}
\hline & $\begin{array}{c}\text { Neutron flux } \\
\text { accuracy }\end{array}$ & \multicolumn{2}{c}{ Reaction rate accuracy } \\
\cline { 3 - 4 } & ${ }^{54} \mathrm{Fe}(\mathrm{n}, \mathrm{p}){ }^{54} \mathrm{Mn}$ & ${ }^{63} \mathrm{Cu}(\mathrm{n}, \alpha){ }^{60} \mathrm{Co}$ \\
\hline MCNP & 1.08 & 1.00 & 0.96 \\
DORT & 1.25 & 1.31 & 1.17 \\
\hline
\end{tabular}

\subsubsection{Neutron data library and neutron energy spectrum}

As shown in Table 3, it is found that the neutron flux calculated by MCNP with HHMCM is more precise than by DORT which is about $30 \%$ larger than the measurement. Of course, an applicability of simulating realistic structures is one of the reason of the high accuracy calculation using MCNP with HHMCM, but there may be other reasons; neutron energy group structure, nuclear data library and neutron spectrum as mentioned at the beginning of this article. These will be examined with the calculation conditions shown in Table 4, but these sensitivity analyses were performed with a simplified model which was consisted of a cylindrical and homogeneous core with a shroud and a 
RPV for both DORT and MCNP.

Case 1 in Table 4 is the same condition of the nuclear data library and neutron spectrum used for the calculation by DORT as shown in Table 1, and Case 2 is the same condition used for the previous calculation by MCNP.

A difference in neutron energy group structure was examined by a sensitivity analysis with MCNP calculations. The difference between Case 2 and Case 3 shows the difference between neutron energy group structures, and the result of using continuous energy group structure is smaller than using the discrete one. The difference between Case 2 and Case 4 shows the difference between both the neutron energy structures and the neutron spectrum, and the result of using both continuous energy nuclear data and neutron spectrum is smallest among case 2 4. Therefore, as the result of using DORT is about 30\% larger than the measurement as shown in Table 3, the calculation of both the continuous energy nuclear data and neutron spectrum was expected to achieve a more precise result than the DORT calculation. In addition, although Case 4 and Case 5 have a small difference in the results, the calculation with ENDF/B-VII [2] was expected to have better accuracy than with JENDL-J33 for this work. Based on the difference between Case 1 and Case 4, an attempt to model the core by HHMCM might redound to the accuracy in the previous section.

Table 4. The conditions and the results of sensitivity analysis

\begin{tabular}{c|c|cccc}
\hline Case & 1 & 2 & 3 & 4 & 5 \\
\hline Code & DORT & \multicolumn{4}{|c}{ MCNP } \\
\hline $\begin{array}{c}\text { Nuclear } \\
\text { data } \\
\text { library }\end{array}$ & $\begin{array}{c}\text { MATXSLIB } \\
\text {-J33 base } \\
\text { Discrete }\end{array}$ & $\begin{array}{c}\text { ENDF/B- } \\
\text { VII:0.x } \\
\text { Continuous }\end{array}$ & $\begin{array}{c}\text { MENDF5 } \\
\text { [iscrete }\end{array}$ & $\begin{array}{c}\text { ENDF/B- } \\
\text { VII:0.x }\end{array}$ & $\begin{array}{c}\text { FXLIB-J33 } \\
\text { [4] }\end{array}$ \\
\hline $\begin{array}{c}\text { Neutron } \\
\text { spectrum }\end{array}$ & $\begin{array}{c}\text { U-235 } \\
\text { Discrete }\end{array}$ & $\begin{array}{c}\text { Watt } \\
\text { spectrum } \\
\text { Continuous }\end{array}$ & $\begin{array}{c}\text { U-235 } \\
\text { Discrete }\end{array}$ & $\begin{array}{c}\text { U-235 } \\
\text { Discrete }\end{array}$ & $\begin{array}{c}\text { Watt } \\
\text { spectrum } \\
\text { Continuous }\end{array}$ \\
\hline $\begin{array}{c}\text { Neutron } \\
\text { flux } \\
\left(\mathrm{n} / \mathrm{cm}^{2}\right.\end{array}$ & $2.0 \mathrm{E}-11$ & $1.7 \mathrm{E}-11$ & $3.3 \mathrm{E}-11$ & $2.2 \mathrm{E}-11$ & $1.9 \mathrm{E}-11$ \\
$/ \mathrm{sec})$ & & & & & \\
\hline
\end{tabular}

\subsubsection{Calculation time saving}

As mentioned at the beginning of this article, if geometry of a source region is too complicated, MCNP requires a huge computing time for the source position sampling procedure. To achieve a good balance between a higher accuracy and a shorter calculation time, HHMCM and the confined area were used as mentioned above. Here, how much time was saved on the calculation by MCNP with HHMCM in Figure 3 will be estimated in this section.

It took 40 hours to calculate the neutron flux by MCNP with HHMCM with a single CPU personal computer. In particular, for the most peripheral bundles simulating the heterogeneous structure, it took 0.3 hours to calculate per one bundle until FSD fell below 10\% [2]. And, for the next to the most peripheral bundles, it took 0.8 hours and was 2.8 times longer than for the most peripheral bundle. Based on this trend if all the fifty-two bundles in Figure 3 were simulated as the heterogeneous bundle structure, it might take 362 hours to calculate. Therefore, it is found that HHMCM might be able to reduce the calculation time to about $1 / 9$.

\section{Conclusion}

The calculation of the fast neutron flux at the RPV inner surface by MCNP with HHMCM had the higher accuracy of approximately $8 \%$ than that by DORT which obtained the accuracy of approximately $25 \%$ in the reference analysis, and the accuracy was far better than the recommended value $20 \%$ [3]. Furthermore, the calculation accuracy of the reaction rates could agree within 4\%. So using MCNP with HHMCM can estimate the fast neutron flux directly and has no use for the correction by the measurement value of the reaction rates like the calculation of using DORT for the surveillance program. In addition, HHMCM could save the computing time compared with the full heterogeneous core model. Therefore we conclude that the calculation of using MCNP with HHMCM is a good way to achieve a high accuracy and to save the computing time for the neutron flux estimation for the RPV surveillance program.

In this work, the core conditions; the neutron generation distribution, the void distribution and the fuel bundle types, were treated not by each bundle but by the region shown in Figure 1. However, if the neutron flux at a more close point to the core is estimated, it might be influenced from these conditions and not be estimated with high accuracy. These conditions might be estimated to understand how much detail they have to be simulated in the future.

\section{References}

[1] F.R. Mynatt and W.A. Rhoades, The DOT-III Two Dimensional Discrete Ordinates Transport Code, ORNL/TM-4280(1973).

[2] X-5 Monte Carlo Team, MCNP - A General Monte Carlo N-Particle Transport Code, Version 5 Volume I: Overview and Theory, LA-UR-03-1987, Revised 2/1/2008.

[3] U.S.Nuclear Regulatory Commission, Regulatory guide 1.190 , calculation and dosimetry methods for determining pressure vessel neutron flux, March 2001, Revision 0.

[4] K. Kosako, N. Yamano, T. Fukahori, K. Shibata and A. Hasegawa, The libraries FSXLIB and MATXSLIB based on JENDL3.3, JAERI-Data/Code 2003-011, 2003. 